\title{
Primary lymphoedema when found with distichiasis of the type defined as bilateral hyperplasia by lymphography
}

\author{
R F D A LE \\ From St Thomas's Hospital, London SE1.
}

SUMMARY The occurrence of primary lymphoedema with distichiasis is well recognised, but thä the form of lymphoedema appears restricted to bilateral hyperplasia, as defined by lymphography has not been reported.

Bilateral hyperplasia is an uncommon form of lymphoedema. Lymphography shows abundant and dilated lymphatics occurring in both lower limbs and the thoracic duct is either absent or, if present, is obstructed or deformed. ${ }^{1}$ It is the only form of lymphoedema described in association with other congenital abnormalities; $16 \%$ of cases have been noted to have congenital heart disease. ${ }^{2}$

Distichiasis, a double row of eyelashes, is a rare condition due to a dominant gene, and its association with lymphoedema has been reported previously. Neel and Schull ${ }^{3}$ point out that this association could be due to the action of a single gene with multiple effects or to close linkage of two different genes.

\section{Case reports}

A recent survey of 725 patients with primary lymphoedema who had attended St Thomas's Hospital revealed five cases with distichiasis. Lymphography had been performed in these five patients and bilateral hyperplasia was shown in each of them.

Bilateral hyperplasia was found in only $30(6 \%)$ of the 475 patients who had been investigated by lymphography, as defined by Kinmonth and Wolfe. ${ }^{4}$ Of these, $11(36 \%)$ had a positive family history and three had both distichiasis and bilateral hyperplasia.

The three patients with both conditions were from two pedigrees. Fig 1 shows the family with two patients (III.1 and IV.2) in which they were the only members known to have distichiasis, though I.2, II.1, II.3, and II.6 had lymphoedema. Fig 2 shows the

Received for publication 26 October 1984

Revised version accepted for publication 22 November 1985. third index patient (III.2) from a family where $a^{\frac{0}{7}}$ uncle (II.2) also had distichiasis. The patient's fathe $\overrightarrow{\mathrm{C}_{0}}$ as well as II.3, II.4, and III.1, had lymphoedema bưf not distichiasis.

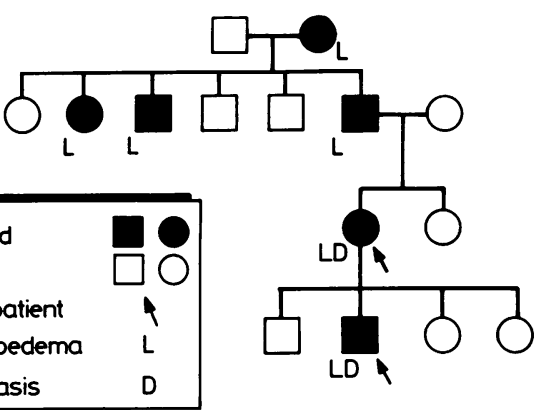

FIG 1 Pedigree of family with two index patients.

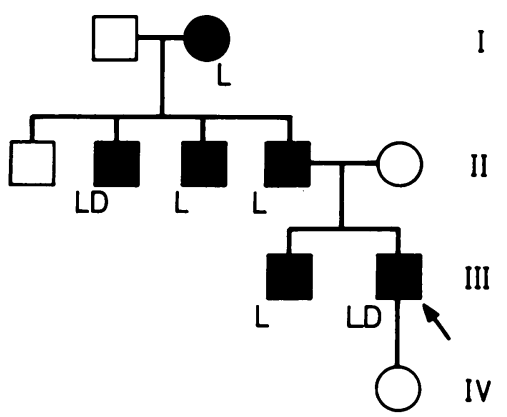

FIG 2 Pedigree of family with one index patient. 


\section{Discussion}

Lymphoedema, when inherited, develops due to the action of a dominant autosomal gene ${ }^{5}$ with incomplete penetrance of about $50 \%$. There is sex influence with a higher proportion of females developing lymphoedema $(\mathrm{M}: \mathrm{F}=0 \cdot 4)$. In the small number of patients with bilateral hyperplasia the sex influence seems to be reversed, with males at greater risk than females $(\mathrm{M}: \mathrm{F}=1 \cdot 7)$.

The possibilities that could explain the occurrence of distichiasis with bilateral hyperplasia are (1) that there is linkage of two genes, (2) that there are two pleiotropic effects of the same incompletely penetrant gene with variable expression, or (3) that this is simply association.

Distichiasis is a rare condition and it does occur separately from lymphoedema. Fox surveyed published reports in $1962^{6}$ and found 78 cases described. $\mathrm{He}$ found a strong hereditary influence without any sex predeliction but did not mention any connection with lymphoedema. McKusick ${ }^{7}$ quotes Maumenee (personal communication, 1982) that it is only where lymphoedema is also present that distichiasis is inherited. Published reports are confusing and this may be due to the variability of expression of the two traits, particularly the late onset of lymphoedema in many cases.

Bilateral hyperplasia is a rare form of a rare condition and it is unlikely that distichiasis would only appear in this small group from the effect of linkage. There is no reason to think that the combination has any effect on survival and linkage disequilibrium cannot explain the findings.
Association is an unlikely explanation for the two conditions to appear together as they are both rare and one would have expected distichiasis to occur with other forms of lymphoedema.

\section{Conclusion}

The appearance of distichiasis with primary lymphoedema is restricted to those with bilateral hyperplasia of the lymphatics. If distichiasis is seen in someone with lymphoedema, it is likely that patient has bilateral hyperplasia rather than other forms of lymphoedema. Distichiasis and bilateral hyperplasia appear to be inherited through the action of an incompletely penetrant gene with two pleiotropic effects and variable expression.

\footnotetext{
References

' Kinmonth JB. The lymphatics. London: Arnold, 1982:102.

2 Corbett CRR, Dale RF, Coltart DJ, Kinmonth JB. Congenital heart disease in patients with primary lymphoedemas. Lymphology 1982;15:85-90.

${ }^{3}$ Neel JV, Schull WJ. Human heredity. Chicago: University of Chicago Press, 1954:50-1.

${ }^{4}$ Kinmonth JB, Wolfe JH. Fibrosis in the lymph nodes in primary lymphoedema. Ann $R$ Coll Surg Engl 1980;62:344-54.

5 Dale RF. The inheritance of primary lymphoedema. $J$ Med Genet 1985;22:274-8.

${ }^{6}$ Fox SA. Distichiasis. Am J Ophthalmol 1962;53:14-8.

7 McKusick VA. Mendelian inheritance in man. 6th ed. Baltimore: Johns Hopkins University Press, 1983:140.
}

Correspondence and requests for reprints to $\mathrm{Mr}$ R F Dale, FRCS, Senior Surgical Registrar, Addenbrooke's Hospital, Hills Road, Cambridge CB2 2QQ.

\section{Correction}

In the paper 'Carrier detection and prenatal diagnosis in $\mathrm{X}$ linked muscular dystrophy using restriction fragment length polymorphisms' by Lindlö $e t$ al, published in the December 1986 issue of the Journal ( $J$ Med Genet 1986;23:560-72), an error occurred in fig 1. The pERT87 alleles underneath III.1 in family 5 should read S2, F2 and not S1, F2. 